\title{
PERSPECTIVE OPEN Manipulation of charge transport in thermoelectrics
}

\author{
Xinyue Zhang ${ }^{1}$ and Yanzhong Pei ${ }^{1}$
}

While numerous improvements have been achieved in thermoelectric materials by reducing the lattice thermal conductivity $\left(K_{\mathrm{L}}\right)$, electronic approaches for enhancement can be as effective, or even more. A key challenge is decoupling Seebeck coefficient $(S)$ from electrical conductivity $(\sigma)$. The first order approximation - a single parabolic band assumption with acoustic scattering - leads the thermoelectric power factor $\left(S^{2} \sigma\right)$ to be maximized at a constant reduced Fermi level $(\eta \sim 0.67)$ and therefore at a given $S$ of $\sim 167 \mu \mathrm{V} / \mathrm{K}$. This simplifies the challenge of maximization of $\sigma$ at a constant $\eta$, leading to a large number of degenerate transport channels (band degeneracy, $N_{\mathrm{v}}$ ) and a fast transportation of charges (carrier mobility, $\mu$ ). In this paper, existing efforts on this issue are summarized and future prospectives are given.

npj Quantum Materials (2017)2:68; doi:10.1038/s41535-017-0071-2

\section{INTRODUCTION}

Thermoelectricity

Thermoelectric (TE) technique, which directly converts heat into electricity and vise-versa, has attracted increasing attention for power generation and refrigeration. The efficiency is largely determined by materials' TE figure of merit, $z T=S^{2} \sigma T /\left(\kappa_{\mathrm{E}}+\kappa_{\mathrm{L}}\right)$, where $S, \sigma, T, \kappa_{\mathrm{E}}$, and $\kappa_{\mathrm{L}}$ represent the Seebeck coefficient, electrical conductivity, absolute temperature, and electronic and lattice contributions to the total thermal conductivity $\kappa$, respectively. $^{1}$

Proven thermal strategy for enhancing $z T$ is to minimize $\kappa_{\mathrm{L}}$ through microstructure engineering for enhancement of the scattering of phonons $\mathrm{s}^{2-6}$ and through intrinsically low lattice thermal conductivity due to various mechanisms. ${ }^{7-11}$ While the remaining three electronic parameters $S, \rho$, and $\kappa_{\mathrm{E}}$ are strongly coupled with each other through the carrier concentration $(n)$, band structure and scattering, leaving a key challenge to decouple these parameters for increasing power factor.

In order to decouple these parameters effectively, the essence of Seebeck coefficient is important to re-emphasize. Considering the charge transport in a parabolic band with acoustic scattering, as which most of thermoelectrics can be approximated, ${ }^{12}$ Seebeck coefficient only depends on the reduced Fermi level $(\eta)$ and power factor $\left(S^{2} \sigma\right)$ maximized at a constant $\eta \sim 0.67 .^{13,14}$ This means a maximal power factor can only be realized at such a constant $\eta$, leading the Seebeck coefficient to be fixed at $\sim 167 \mu \mathrm{V} / \mathrm{K}$. Therefore, the decouple between $S$ and $\sigma$, for an increase in maximal power factor can actually be simplified as the increase of $\sigma$ at the optimal $\eta \sim 0.67$. It should be noted that a constant $\eta$ actually requires a strong temperature and density of states (DOS) mass $\left(m_{\mathrm{d}}{ }^{*}\right)$-dependent carrier concentration $\left[n_{\mathrm{opt}} \sim\left(m_{\mathrm{d}}{ }^{*} T\right)^{1.5}\right] .{ }^{15}$

Both large number of transporting bands and fast transportation of carriers contribute to increasing $\sigma$, at a given $\eta$. A large band degeneracy $N_{v}$ offers multiple transporting channels thus benefit $\sigma$. And the fast transportation of carriers, meaning a high carrier mobility $\mu$, requires a small effective mass as well as a weak scattering of carriers.
Essence of seebeck coefficient

Based on Boltzmann transport theory, the Seebeck coefficient for conduction by a single type of carriers (either electrons or holes) is given by: ${ }^{13}$

$s=\frac{1}{T} \frac{\int_{0}^{\infty} g(E) \tau_{e} E\left(E-E_{\mathrm{F}}\right) \frac{\mathrm{d} f_{0}(E)}{\mathrm{d} E} \mathrm{~d} E}{e \int_{0}^{\infty} g(E) \tau_{e} E \frac{\mathrm{d} f_{0}(E)}{\mathrm{d} E} \mathrm{~d} E}$

Here, - and + stands for conduction and valence band, respectively. $E_{\mathrm{F}}, f_{0}, e$ and $\tau_{\mathrm{e}}$ represent the Fermi level, the Fermi distribution function, the electron charge and the relaxation time for charge carriers, respectively. Only carriers with an energy in the vicinity of the Fermi level effectively contribute to the charge transport, determined by $\mathrm{d} f_{o}(E) / d E$. Since the denominator of Eq. 1 stands for the electrical conductivity, the sign and quantity of Seebeck coefficient largely depend on the numerator of Eq. 1. Schematic plots of the integrand function $\left[g(E) \tau_{e} E\left(E-E_{\mathrm{F}}\right) \frac{\mathrm{d} f_{0}(E)}{\mathrm{d} E}\right]$ in the numerator of Eq. 1 versus Fermi energy are shown in Fig. 1.

It is seen that partial contributions $\left(S_{1}\right.$ and $S_{2}$, shaded areas) to the net Seebeck coefficient, respectively due to the carriers with energy above or below the Fermi level, have opposite signs.

$$
\begin{aligned}
& S_{1}=\int_{E_{\mathrm{F}}}^{\infty} g(E) \tau_{e} E\left(E-E_{\mathrm{F}}\right) \frac{\mathrm{d} f_{0}(E)}{\mathrm{d} E} \mathrm{~d} E>0 \\
& S_{2}=\int_{\infty}^{E_{\mathrm{F}}} g(E) \tau_{e} E\left(E-E_{\mathrm{F}}\right) \frac{\mathrm{d} f_{0}(E)}{\mathrm{d} E} \mathrm{~d} E<0
\end{aligned}
$$

Using the reduced Fermi energy $\eta=E / k_{\mathrm{B}} T$, Eq. 1 can be simplified as Eq. 4 in terms of Fermi-Dirac integrals for charge transport by a parabolic band. ${ }^{11}$

$S(\eta)=\mp \frac{k_{\mathrm{B}}}{e}\left[\frac{(r+5 / 2) F_{(r+3 / 2)}(\eta)}{(r+3 / 2) F_{(r+1 / 2)}(\eta)}-\eta\right]$

The scattering parameter $r$ in this equation is the exponent of $\tau_{\mathrm{e}}$ versus energy via $\tau_{0} E^{r}$, where $\tau_{0}$ is the relaxation time for nondegenerate carriers. This clearly reveals that $S$ depends only on $\eta$, at a given type of scattering. Since the scattering of carriers is

\footnotetext{
${ }^{1}$ Interdisciplinary Materials Research Center, School of Materials Science and Engineering, Tongji University, 4800 Caoan Road, Shanghai 201804 , China
} Correspondence: Yanzhong Pei (yanzhong@tongji.edu.cn) 


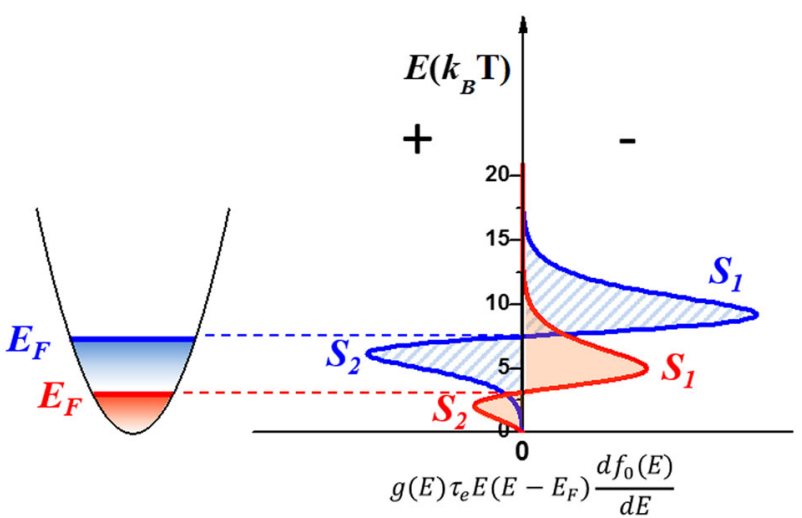

Fig. 1 Essence of Seebeck Coefficient. Schematic plots of the integrand function in the numerator of Eq. 1 versus reduced Fermi energy. Carriers with energy above or below the Fermi level oppositely $\left(S_{1}\right.$ and $S_{2}$, shaded areas) contribute to net Seebeck coefficient

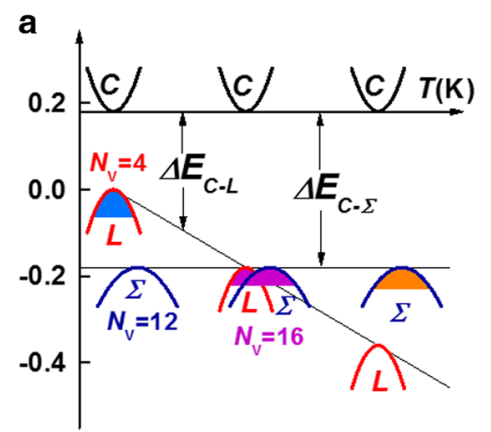

b

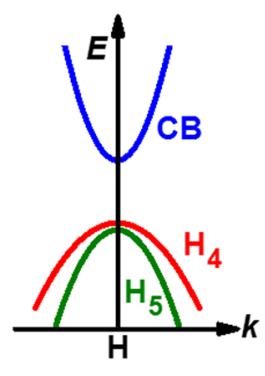

Fig. 2 Band convergence and nestification. a Schematic diagram of temperature-induced band convergence in $\mathrm{PbTe}$; $\mathbf{b}$ Inherent band nestification in elemental tellurium, enabling a large number of effective band valley degeneracy

dominated by acoustic phonons in most of known good thermoelectrics when $z T$ is high, $^{16}$ this ensures an observation in thermoelectrics a decrease in $S$ when carrier concentration increases (high $\eta$ ) at a given temperature, while an increase in $S$ as temperature rises (low $\eta$ ) at a given carrier concentration.

\section{PROVEN STRATEGIES}

Large band degeneracy

Due to the monotonous dependence between $S$ and $\eta$, the challenge to decouple $S$ and $\sigma$ can be simplified to a maximization of $\sigma$ at the optimal $\eta$ peaking power factor. A large $N_{v}$ is clearly beneficial because large $N_{v}$ offers multiple conducting channels for high electrical conductivity, while equivalently overlapped bands for a large $N_{v}$ induce no effects on $S$ as $\eta$ is given. $N_{v}$ increases effectively when multiple bands have the same or comparable energy within a few $k_{\mathrm{B}} T$, including orbital degeneracy and valley degeneracy. ${ }^{12}$

Converging different bands, which locate at different positions in the reciprocal space, is proven to be successful for increasing $N_{\mathrm{v}}$, therefore $z T$, in compounds with multiple bands, such as PbTe (Fig. 2a), ${ }^{17} \mathrm{SnTe}^{18-21} \mathrm{CoSb}_{3}{ }^{22} \mathrm{SnSe}^{23}$ half-Heusler $(\mathrm{HH})^{24}$ and $\mathrm{Mg}_{3} \mathrm{Sb}_{2} .{ }^{25}$ It is worth noting that the convergence temperature for PbTe used to be believed to be around $450 \mathrm{~K} .^{17,26,27}$ However, recent investigation and data interpretation have indicated that the convergence temperature should be higher. ${ }^{28,29}$ Nevertheless, all existing studies consistently show that the valence bands in $\mathrm{PbTe}$ tend to converge at high temperatures, which helps understand the high $z T$ observed in this material at high temperatures.

Convergence of split bands can be realized through crystal structure design as well. When chalcopyrite compounds are engineered to have a pseudo-cubic structure, a realization of high band degeneracy thus high TE performance was reported. ${ }^{30}$ Extend this concept of converging split bands to layered Zintl phase TE materials, high orbital degeneracy is realized by a design of chemical composition. ${ }^{8}$ In addition, structure transition has also been utilized for band convergence, this is typified by the recent $\mathrm{Bi}_{2-x} \mathrm{Sb}_{x} \mathrm{Se}_{3}$ alloys, ${ }^{31}$ where the location of conduction bands can be engineered for a large $N_{\mathrm{v}}$.

Alternative to converging bands having different energies, many semiconductors offer an inherent band nestification, ${ }^{32}$ equally enabling a large number of effective band degeneracy. Guided by this concept, a $z T$ as high as unity is achieved in elemental Tellurium (Fig. 2b), ${ }^{33}$ filling the gap of high performance elemental thermoelectrics at 300-700 K.

\section{Low effective mass}

Under an approximation of single parabolic band conduction with acoustic scattering, it is known that maximal power factor can be characterized by the weighted mobility $\left(m_{\mathrm{d}}{ }^{* 3 / 2} \mu_{0}\right){ }^{13,34}$ where the density-of-states effective mass $m_{\mathrm{d}}{ }^{*}=N_{\mathrm{v}}{ }^{2 / 3} m_{\mathrm{b}}{ }^{*}$ and the nondegenerate mobility $\mu_{0} \propto 1 /\left(m_{1}^{*}\left(m_{\mathrm{b}}^{*}\right)^{3 / 2} \equiv^{2} T^{3 / 2}\right)$ with $m_{\mathrm{b}}^{*}$ as the band effective mass. ${ }^{35}$ This leads the maximal power factor to be proportional to $N_{\mathrm{v}}$ but inversely proportional to inertial mass $\left(m_{1}^{*}\right)$ and deformation potential coefficient (E) via $S^{2} \sigma \propto\left(m_{\mathrm{d}}{ }^{* 3 / 2} \mu\right) \propto$ $N_{\mathrm{v}} m_{1}{ }^{-1} \Xi^{-2}$. 36

A successful demonstration of a low effective mass for a high $z T$ is typified by $n$-type PbTe. ${ }^{37}$ A lower $m_{1}^{*}$ in I-doped samples leads to a higher mobility thus higher $z T$, as compared to that of Ladoped ones. As for $\mathrm{HH}$ compounds $\left(m_{\mathrm{d}}{ }^{*}\right.$ of $2 m_{\mathrm{e}} \sim 10 m_{\mathrm{e}}$ ) having bands much heavier than traditional thermoelectrics $\left(m_{\mathrm{d}}{ }^{*}\right.$ of $\left.0.1 m_{\mathrm{e}} \sim 1.0 \mathrm{~m}_{\mathrm{e}}\right){ }^{24}$ reduction of effective mass for increasing the mobility offers great opportunities for $z T$ enhancements. This is realized in FeNbSb, ${ }^{38}$ where a significant decrease of $m_{\mathrm{b}}{ }^{*}$ can be found from $2.5 m_{\mathrm{e}}$ for p-type $\mathrm{FeV}_{0.6} \mathrm{Nb}_{0.4} \mathrm{Sb}$ to $1.6 \mathrm{~m}_{\mathrm{e}}$ for FeNbSb, leading to a higher carrier mobility and a higher $z T$. More recently, excellent TE performance is predicted in novel materials $\mathrm{Cu}_{3} \mathrm{MCh}_{4}$ $(\mathrm{M}=\mathrm{V}, \mathrm{Nb}, \mathrm{Ta} ; \mathrm{Ch}=\mathrm{Se}, \mathrm{Te})$, and higher $z T$ predicted for p-type is mainly due to a smaller effective mass of holes $\left(0.3 m_{\mathrm{e}} \sim 0.5 m_{\mathrm{e}}\right)$ than that of electrons $\left(2 m_{\mathrm{e}} \sim 3 m_{\mathrm{e}}\right)$. $^{39}$

More recently, the decrease of the chemical bond iconicity (increase of the bond covalency) has been reported to be effective on decreasing the carrier effective mass, which leads to a high carrier mobility and thereby a high power factor in $\mathrm{BiCuSeO}{ }^{40}$ This approach has been demonstrated in CdSb as well, in which the anisotropic multicenter bonds lead to a low overall ionicity and thus a high carrier mobility. ${ }^{41}$

\section{Low deformation potential coefficient}

Besides the low inertial effective mass, a low deformation potential coefficient $(\Xi)$ is also expected for high mobility. A low 三 corresponds to a weak scattering of carriers by acoustic phonons. A successful demonstration on the importance of low $\Xi$ is achieved in n-type PbSe. ${ }^{42}$ Despite a low $N_{\mathrm{v}}$ of 4 for the conduction band as compared to a total $N_{v}$ of $>12$ at high temperatures for the valence bands, the $z T$ of n-type PbSe is actually comparable to that of $p$-type. ${ }^{42}$ According to the detailed measurements on carrier transport properties and modeling by a single Kane band (SKB) model, the band effective mass $\left(m_{\mathrm{b}}{ }^{*}\right)$ and $N_{\mathrm{v}}$ are about the same for both conduction and valence bands at $L$ point, and their nearly the same sound velocities confirm the unchanged elastic modulus. However, the SKB model shows different deformation potential coefficients (E) for p-type and ntype $\mathrm{PbSe}$ (35 eV for p-type $\mathrm{PbSe}$ and $25 \mathrm{eV}$ for n-type $\mathrm{PbSe}$ ), 
which means a weaker scattering of electrons as compared with that of holes under the same degree of vibrational deformation, leading to a higher mobility and thereby a high $z T$ in n-type as well. ${ }^{42}$ The benefit of weak scattering by acoustic phonons (low $\equiv$ ) for high mobility and thus $z T$, has also been observed in MgAgSb. ${ }^{43}$ Unfortunately, factors directly affect deformation potential coefficient remain not fully clear, and can be a future direction.

\section{Modulation doping}

Many TE materials require a heavy doping to locate the optimal carrier concentration maximizing $z T^{44-46}$ Largely resulting from the required high $N_{\mathrm{v}}$, such an optimal carrier concentration can be as high as $10^{21} \mathrm{~cm}^{-3}$, therefore the correspondingly required high concentration chemical dopants can act as coulombic scattering centers for carriers. This is important for thermoelectrics since the effective mass is usually small, leading to a strong scattering by ionized impurities. lonized impurity scattering has been considered as an effective way to enhance the Seebeck coefficient. ${ }^{47,48}$ However, this enhancement is realized at an expense of reducing the carrier mobility. The resultant tradeoff between the reduction in electrical conductivity and the enhancement in Seebeck coefficient, leads a net increase in power factor to be achieved in materials with a careful design of doping for manipulating the degree of ionized impurity scattering incorporating with the inherent acoustic scattering.

Concept of modulation doping ${ }^{49}$ was proposed to weaken the ionized impurity scattering for a high mobility, while fulfills the requirement of sufficient carriers in the material. A typical demonstration is undoped Si-Ge TE alloys with nano-inclusions of heavily doped $\mathrm{Si}$, where charge carriers can be separated from nanoparticles into matrix, leaving ionized nanoparticles instead of randomly distributed ionized point defects. Therefore, the charge carriers only experience scattering by ionized nanoparticles distributed much further apart in composites as compared to that by random ionized point defects in alloys, leading to a reduction of scatter rate for a high mobility in nano-composites. ${ }^{49}$

\section{PERSPECTIVES}

Directional lattice distortion

Many materials having a large $N_{\mathrm{v}}$ due to the existence of multiple bands locating at different low symmetry points in the Brillouin zone. Since different locations in reciprocal space correspond to different directions in the real space, the required strain for tuning the band energy individually is highly directional. This is unfortunately impossible in cubic compounds, because an isotropic strain is only allowed through lattice expansion or contraction. Therefore, this leads to a strategy of directional lattice distortion to manipulate the energy of a certain band (bands) for a larger band degeneracy.

Taking GeTe as an example, which crystalizes in a rhombohedral structure, its lattice can be approximated as a slight distorted $\mathrm{NaCl}$ structure along the [111] direction. Since this direction corresponds to the $L$ band, such a directional lattice distortion leads to a switch in energy between $L$ and $\Sigma$ valence bands, enabling the first valence band $(\Sigma)$ of a higher band degeneracy than that of cubic analog compounds. ${ }^{50}$ This strategy would open new possibilities for a large $N_{\mathrm{v}}$ in low-symmetry materials.

\section{Dielectric engineering}

When the dielectric constant is large, a strong dielectric screening will be obtained, therefore the scattering of charge carriers due to ionized impurities would be significantly weak. This motivates a strategy of dielectric engineering ${ }^{51}$ for a high mobility even in a material with high concentration of ionized impurities. Such an effect has been realized for increasing the mobility of 1D and 2D semiconductors. It is found that a creation of dielectric environments surrounding the low dimensional semiconductors, through such as coating with high- $K$ dielectrics, strongly weakens the scattering of carriers due to charged impurities and thus leads to an improvement in mobility. ${ }^{51}$ This strategy is not widely used in bulk TE materials to date, but it in principle offers inspirations for future mobility engineering in bulk TE materials and semiconductors in general.

\section{Carrier injection}

Since most of known thermoelectrics are narrow-gap semiconductors, the broadening of Fermi distribution at high temperatures as well as insufficient doping would lead to a coexistence of both electrons and holes. When the both exist in a material, the flow directions of these two types of carriers are the same and both follow the direction of heat flow, leading to a strong compensation to thermopower (Fig. 3a) and an increase in electronic thermal conductivity. Therefore, not only the solubility of dopants but also the resulting ionized impurity scattering limit the realization of full potential of a material for TE applications.

Instead of chemical doping, an electric field enables injection/ removal of carriers (Fig. 3b) and thus a continuous control of carrier concentration. Such an electrostatic doping effect does not introduce additional scattering due to ionized impurities and validates materials that are difficult to be doped as effective thermoelectrics, both of which are beneficial for improving both existing and new thermoelectrics. It is revealed that an optimization of carrier concentration through electrostatic doping leads to a power factor in ultrathin $\mathrm{WSe}_{2}$ single crystals as high as $40 \mu \mathrm{W} /$ $\mathrm{cm}-\mathrm{K}^{2}{ }^{52}$ It is worth pointing out that electrostatic doping may have drawbacks on the possible leakage of heat/current, the a

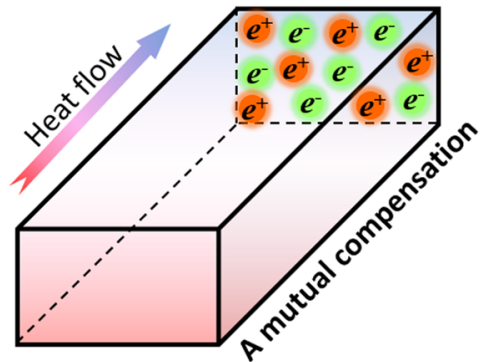

Coexistence of Electrons and holes b

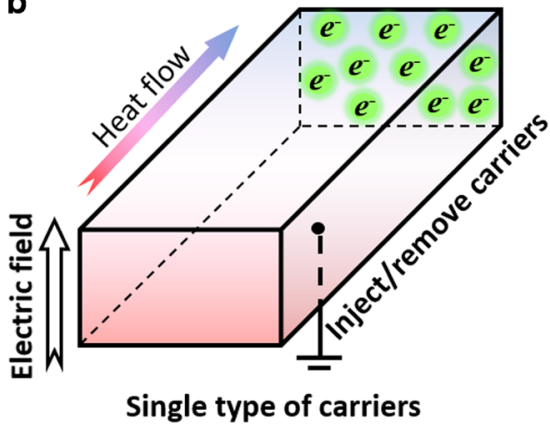

Fig. 3 Electrostatic doping. a When electrons and holes coexist, the flow directions of these two types of carriers are the same and both follow the direction of heat flow, which would lead to a strong compensation to thermopower; $\mathbf{b}$ injection/removal of carriers through electric field enables a continuous control of carrier concentration 


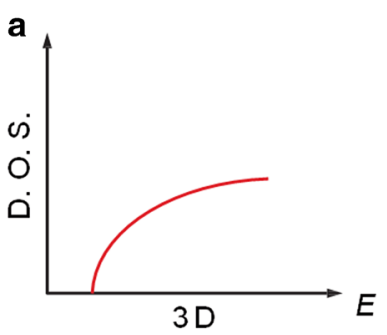

Bulk Semiconductor

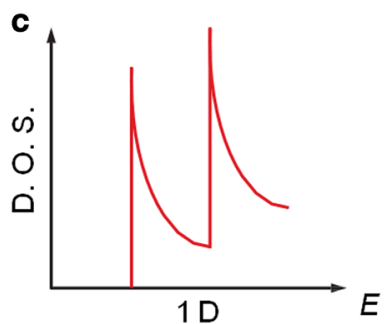

Quantum Wire
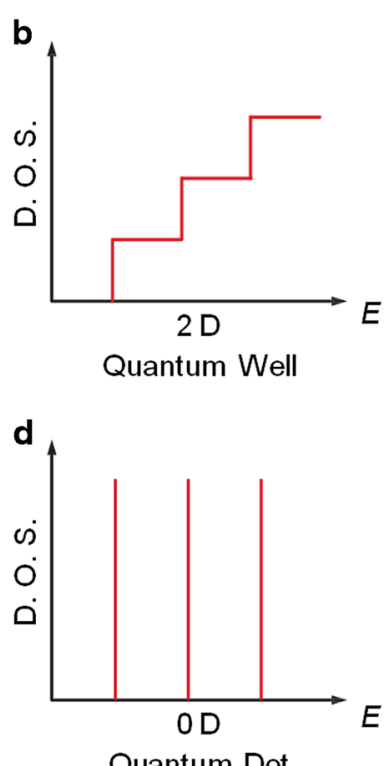

Fig. 4 Quantum confinement effect on electronic density of states. Electronic density of states for a a $3 D$ semiconductor, b a 2D quantum well, $\mathbf{c}$ a $1 \mathrm{D}$ nanowire or nanotube and $\mathbf{d}$ a $0 \mathrm{D}$ quantum dot. (Adapted from ref. 56)

complexity of device structure as well as the energy consumption of external electric field.

In addition, utilizing the ferromagnetic-paramagnetic phase transition of the impurity phase in a TE composite, charge carriers localized in ferromagnetic impurity phase due to magnetoelectric interactions can be injected into the matrix phase at temperatures higher than the Curie temperature of the magnetic impurity phase. $^{53}$ This would enable a control on the effective carrier concentration of thermoelectrics. The above concepts can expand the category of TE materials and offers additional degree of freedom for performance manipulation.

Quantum confinement and other strategies

In addition to the strategies of enhancing electrical conductivity at given $\eta$, other strategies alternatively focus on the enhancement of Seebeck coefficient, many of which takes the advantages of the low dimensional features. About 20 years ago, the concept of quantum confinement was proposed by Hicks and Dresselhaus, offering additional degrees of freedom for enhancing the TE performance because of the strong dependence between electronic DOS and dimensionality ${ }^{54-56}$ (Fig. 4). For example, when the dimensionality of a $3 \mathrm{D}$ material is decreased to $2 \mathrm{D}$ quantum wells, the new variable of layer thickness becomes available for the control of material properties. ${ }^{55}$ This concept opens new possibility for manipulating charge transport in low dimensional materials.

Since the low dimensionality opens new possibilities for obtaining a high power factor, theoretical studies suggest topological insulators as promising thermoelectrics. ${ }^{57}$ It is shown that the unique electronic structure of the boundary (surface or edge) states together with bulk states in two-dimensional topological insulators leads to strong size dependence of transport properties, ${ }^{57}$ especially the Seebeck coefficient. Interestingly, some of the features of transport property that usually be observable in low-dimensional materials can even be realized in $3 \mathrm{D}$ materials. For an example, a very high Seebeck coefficient is obtained in a 3D material BiTel, ${ }^{58}$ due to the 2D-like electron DOS resulting from the strong band splitting. Theoretical calculation further reveals that the existence of Rashba spin-split bands leads to an enhancement in the power factor as compared to that of with spin-degenerate bands.

Another concept, energy filtering, has further advanced the potential for low-dimensional structures to obtain a high power factor. ${ }^{59}$ For bulk materials with nanoparticles ${ }^{60}$ or grain-boundary interfaces, ${ }^{61}$ the observed high power factor is usually ascribed to the energy filtering effect. However, it's worth noting that a full realization of energy filtering effect is still a challenge, due to the leakage of technique for a direct observation/characterization as well as an accurate design of energy potential barriers. ${ }^{62}$ These strategies relying on low dimensionality provide new insights for future research directions for thermoelectrics.

\section{SUMMARY}

Since Seebeck coefficient depends only on the reduced Fermi level $\eta$ and the power factor maximized at a constant $\eta$, this simplifies the challenge, of decoupling Seebeck coefficient and electrical conductivity for an enhanced power factor, to the maximization of electrical conductivity at optimal $\eta$. Existing strategies demonstrated including large band degeneracy, low effective mass, low deformation potential coefficient and modulation doping are summarized. Available room for further improvements is discussed as well, with perspective strategies proposed, including directional lattice distortion, dielectric engineering, carrier injection, quantum confinement and other relative strategies. Although these are not widely adopted yet, initial efforts indeed lead to effective improvements on TE performance.

\section{ACKNOWLEDGEMENTS}

This work is supported by the National Natural Science Foundation of China (Grant No. 51422208, 11474219 and 51772215).

\section{AUTHOR CONTRIBUTIONS}

Y.P. advised the conception of this article and oversaw the overall structure of the manuscript. X.Z. drafted the article. All authors contribute to the writing and revision of the manuscript.

\section{ADDITIONAL INFORMATION}

Competing interests: The authors declare that they have no competing financial interests.

Publisher's note: Springer Nature remains neutral with regard to jurisdictional claims in published maps and institutional affiliations.

\section{REFERENCES}

1. Snyder, G. J. \& Toberer, E. S. Complex thermoelectric materials. Nat. Mater. 7, 105-114 (2008)

2. Poudel, B. et al. High-thermoelectric performance of nanostructured bismuth antimony telluride bulk alloys. Science 320, 634-638 (2008).

3. Kim, S. I. et al. Dense dislocation arrays embedded in grain boundaries for highperformance bulk thermoelectrics. Science 348, 109-114 (2015).

4. Chen, Z. et al. Vacancy-induced dislocations within grains for high-performance PbSe thermoelectrics. Nat. Commun. 8, 13828 (2017).

5. Chen, Z. et al. Lattice dislocations enhancing thermoelectric PbTe in addition to band convergence. Adv. Mater. 29, 1606768 (2017).

6. Pei, Y. et al. Interstitial point defect scattering contributing to high thermoelectric performance in SnTe. Adv. Electron. Mater. 2, 1600019 (2016).

7. Morelli, D. T., Jovovic, V. \& Heremans, J. P. Intrinsically minimal thermal conductivity in cubic I-V-VI 2 semiconductors. Phys. Rev. Lett. 101, 035901 (2008).

8. Li, W. et al. Low sound velocity contributing to the high thermoelectric performance of $\mathrm{Ag}_{8} \mathrm{SnSe}_{6}$. Adv. Sci. 3, 1600196 (2016).

9. Liu, H., Shi, X., Xu, F., Zhang, L. \& Zhang, W. Copper ion liquid-like thermoelectrics. Nat. Mater. 11, 422-425 (2012).

10. Lin, S. et al. High thermoelectric performance of $\mathrm{Ag}_{9} \mathrm{GaSe}_{6}$ enabled by low cut-off frequency of acoustic phonons. Joule 1, https://doi.org/10.1016/j.joule. 2017.09.006 (2017). 
11. Zhang, X. et al. Promising thermoelectric $\mathrm{Ag}_{5-\delta} \mathrm{Te}_{3}$ with intrinsic low lattice thermal conductivity. ACS Energy Lett. 2, 2470-2477 (2017).

12. Pei, Y., Wang, H. \& Snyder, G. J. Band engineering of thermoelectric materials. Adv. Mater. 24, 6125-6135 (2012).

13. Goldsmid, H. J. Introduction to Thermoelectricity, 43-62 (Springer, Heidelberg, 2009).

14. Bhandari, C. M. \& Rowe, D. M. in CRC Handbook of Thermoelectrics (ed Rowe, D. M.) Ch. 4, 27-42 (CRC Press, Boca Raton, 1995).

15. loffe, A. F. Semiconductor Thermoelements, and Thermoelectric Cooling. (Infosearch, London, 1957).

16. Ravich, Y. I., Efimova, B. A. \& Smirnov, I. A. Semiconducting Lead Chalcogenides. (Plenum Press, New York, 1970).

17. Pei, Y. et al. Convergence of electronic bands for high performance bulk thermoelectrics. Nature 473, 66-69 (2011).

18. Zheng, L. et al. Interstitial defects improving thermoelectric SnTe in addition to band convergence. ACS Energy Lett. 2, 563-568 (2017).

19. $\mathrm{Li}, \mathrm{W}$. et al. Promoting SnTe as an eco-friendly solution for $\mathrm{p}-\mathrm{PbTe}$ thermoelectric via band convergence and interstitial defects. Adv. Mater. 29, 1605887 (2017).

20. Li, W. et al. Band and scattering tuning for high performance thermoelectric $\mathrm{Sn}_{1-x} \mathrm{Mn}_{x}$ Te alloys. J. Materiomics 1, 307-315 (2015).

21. Li, W. et al. Advances in Environment-Friendly SnTe Thermoelectrics. ACS Energy Lett. 2, 2349-2355 (2017).

22. Tang, Y. et al. Convergence of multi-valley bands as the electronic origin of high thermoelectric performance in $\mathrm{CoSb}_{3}$ skutterudites. Nat. Mater. 14, 1223-1228 (2015).

23. Kurata, K. \& Hirai, T. Semiconducting properties of several $\| I_{B}-\mathrm{V}_{B}-\mathrm{VI}_{B}$ ternary materials and their metallurgical aspects. Solid State Electron 9, 633-640 (1966).

24. Fu, C. et al. Realizing high figure of merit in heavy-band p-type half-Heusler thermoelectric materials. Nat. Commun. 6, 8144 (2015).

25. Zhang, J. et al. Discovery of high-performance low-cost $\mathrm{n}$-type $\mathrm{Mg}_{3} \mathrm{Sb}_{2}$-based thermoelectric materials with multi-valley conduction bands. Nat. Commun. 8 13901 (2017)

26. Andreev, A. A. \& Radionov, V. N. Determination of band structure of lead telluride from measurements of hall effect at high temperatures. Sov. Phys. Semicond. 1, 145-148 (1967)

27. Gibson, A. F. The absorption spectra of single crystals of lead sulphide, selenide and telluride. Proc. Phys. Soc. B 65, 378-388 (1952).

28. Gibbs, Z. M. et al. Temperature dependent band gap in $\mathrm{PbX}(\mathrm{X}=\mathrm{S}, \mathrm{Se}, \mathrm{Te})$. Appl. Phys. Lett. 103, 262109 (2013).

29. Jaworski, C. M. et al. Valence-band structure of highly efficientp-type thermoelectric PbTe-PbS alloys. Phys. Rev. B 87, 045203 (2013).

30. Xiao, Y. et al. Origin of low thermal conductivity in SnSe. Phys. Rev. B 94, 125203 (2016).

31. Wang, $\mathrm{S}$. et al. High thermoelectric performance in Te-free $(\mathrm{Bi}, \mathrm{Sb})_{2} \mathrm{Se}_{3}$ via structural transition induced band convergence and chemical bond softening. Energy Environ. Sci. 9, 3436-3447 (2016).

32. Vurgaftman, I., Meyer, J. R. \& Ram-Mohan, L. R. Band parameters for III-V compound semiconductors and their alloys. J. Appl. Phys. 89, 5815-5875 (2001).

33. Lin, S. et al. Tellurium as a high-performance elemental thermoelectric. Nat. Commun. 7, 10287 (2016)

34. Rowe, D. M. Thermoelectrics Handbook: Macro to Nano. (CRC/Taylor \& Francis, Boca Raton, 2006).

35. Bardeen, J. \& Shockley, W. Deformation potentials and mobilities in non-polar crystals. Phys. Rev. 80, 72-80 (1950).

36. Goldsmid, H. J. Thermoelectric Refrigeration (Plenum Press, New York, 1964).

37. Pei, Y., LaLonde, A. D., Wang, H. \& Snyder, G. J. Low effective mass leading to high thermoelectric performance. Energy Environ. Sci. 5, 7963-7969 (2012).

38. Fu, C., Zhu, T., Liu, Y., Xie, H. \& Zhao, X. Band engineering of high performance ptype FeNbSb based half-Heusler thermoelectric materials for figure of merit $z T>1$. Energy Environ. Sci. 8, 216-220 (2015).

39. Hong, A. J., Yuan, C. L., Gu, G. \& Liu, J. M. Novel p-type thermoelectric materials $\mathrm{Cu}_{3} \mathrm{MCh}_{4}(\mathrm{M}=\mathrm{V}, \mathrm{Nb}, \mathrm{Ta} ; \mathrm{Ch}=\mathrm{Se}, \mathrm{Te})$ : high band-degeneracy. J. Mater. Chem. A 5, 9785-9792 (2017).

40. Ren, G.-K. et al. Enhancing thermoelectric performance in hierarchically structured $\mathrm{BiCuSeO}$ by increasing bond covalency and weakening carrier-phonon coupling. Energy Environ. Sci. 10, 1590-1599 (2017).
41. Wang, S. et al. Anisotropic multicenter bonding and high thermoelectric performance in electron-poor CdSb. Chem. Mater. 27, 1071-1081 (2015).

42. Wang, H., Pei, Y., LaLonde, A. D. \& Snyder, G. J. Weak electron-phonon coupling contributing to high thermoelectric performance in n-type PbSe. Proc. Natl. Acad. Sci. USA 109, 9705-9709 (2012).

43. Liu, Z. et al. Lithium doping to enhance thermoelectric performance of MgAgSb with weak electron-phonon coupling. Adv. Energy Mater. 6, 1502269 (2016).

44. Androulakis, J. et al. Thermoelectric enhancement in PbTe with $\mathrm{K}$ or $\mathrm{Na}$ codoping from tuning the interaction of the light- and heavy-hole valence bands. Phys. Rev. B 82, 115209 (2010).

45. Zhang, X. et al. Thermoelectric properties of GeSe. J. Materiomics 2, 331-337 (2016).

46. Pei, Y., Gibbs, Z. M., Balke, B., Zeier, W. G. \& Snyder, G. J. Optimum carrier concentration in n-type PbTe thermoelectrics. Adv. Energy Mater. 4, 1400486 (2014)

47. Wang, $\mathrm{S}$. et al. On intensifying carrier impurity scattering to enhance thermoelectric performance in $\mathrm{Cr}$-doped $\mathrm{CeyCo}_{4} \mathrm{Sb}_{12}$. Adv. Funct. Mater. 25, 6660-6670 (2015).

48. Dyck, J. S. et al. Thermoelectric properties of then-type filled skutterudite $\mathrm{Ba}_{0.3} \mathrm{Co}_{4} \mathrm{Sb}_{12}$ doped with Ni. J. Appl. Phys. 91, 3698-3705 (2002).

49. Zebarjadi, M. et al. Power factor enhancement by modulation doping in bulk nanocomposites. Nano Lett. 11, 2225-2230 (2011).

50. Li, J. et al. Electronic origin of the high thermoelectric performance of GeTe among the p-type group IV monotellurides. NPG Asia Mater. 9, e353 (2017).

51. Jena, D. \& Konar, A. Enhancement of carrier mobility in semiconductor nanostructures by dielectric engineering. Phys. Rev. Lett. 98, 136805 (2007).

52. Yoshida, M. et al. Gate-optimized thermoelectric power factor in ultrathin WSe single crystals. Nano Lett. 16, 2061-2065 (2016).

53. Zhao, W. et al. Magnetoelectric interaction and transport behaviours in magnetic nanocomposite thermoelectric materials. Nat. Nanotechnol. 12, 55-60 (2017).

54. Hicks, L. D., Harman, T. C., Sun, X. \& Dresselhaus, M. S. Experimental study of the effect of quantum-well structures on the thermoelectric figure of merit. Phys. Rev. $B$ Condens. Matter. 53, R10493-R10496 (1996).

55. Hicks, L. D. \& Dresselhaus, M. S. Effect of quantum-well structures on the thermoelectric figure of merit. Phys. Rev. B 47, 12727-12731 (1993).

56. Mao, J., Liu, Z. \& Ren, Z. Size effect in thermoelectric materials. npj Quant. Mater. 1 , 16028 (2016).

57. Xu, Y., Gan, Z. \& Zhang, S. C. Enhanced thermoelectric performance and anomalous seebeck effects in topological insulators. Phys. Rev. Lett. 112, 226801 (2014)

58. Wu, L. et al. Two-dimensional thermoelectrics with Rashba spin-split bands in bulk BiTel. Phys. Rev. B 90, 195210 (2014).

59. Shakouri, A. \& Bowers, J. E. Heterostructure integrated thermionic coolers. Appl. Phys. Lett. 71, 1234-1236 (1997).

60. Heremans, J. P., Thrush, C. M. \& Morelli, D. T. Thermopower enhancement in PbTe with pb precipitates. J. Appl. Phys. 98, 063703 (2005).

61. Heremans, J. P., Thrush, C. M. \& Morelli, D. T. Thermopower enhancement in lead telluride nanostructures. Phys Rev B 70, 115334 (2004).

62. Zhu, T. et al. Compromise and synergy in high-efficiency thermoelectric materials. Adv. Mater. 29, 1605884 (2017)

(i) Open Access This article is licensed under a Creative Commons adaptation, distribution and reproduction in any medium or format, as long as you give appropriate credit to the original author(s) and the source, provide a link to the Creative Commons license, and indicate if changes were made. The images or other third party material in this article are included in the article's Creative Commons license, unless indicated otherwise in a credit line to the material. If material is not included in the article's Creative Commons license and your intended use is not permitted by statutory regulation or exceeds the permitted use, you will need to obtain permission directly from the copyright holder. To view a copy of this license, visit http://creativecommons. org/licenses/by/4.0/.

(c) The Author(s) 2017 